\title{
Six-pion amplitude
}

\author{
Johan Bijnens $\odot^{*}$ and Tomáš Husek $\oplus^{\dagger}$ \\ Department of Astronomy and Theoretical Physics, Lund University, \\ Sölvegatan 14A, SE 223-62 Lund, Sweden
}

(Received 16 July 2021; accepted 2 September 2021; published 28 September 2021)

\begin{abstract}
Within the framework of the massive $\mathrm{O}(N)$ nonlinear sigma model extended to the next-to-leading order in the chiral counting [for $N=3$ corresponding to the two(-quark)-flavor Chiral Perturbation Theory], we calculate the relativistic six-pion scattering amplitude at low energy up to and including terms $\mathcal{O}\left(p^{4}\right)$. Results for the pion mass, decay constant, and four-pion amplitude in the case of $N$ (meson) flavors at $\mathcal{O}\left(p^{4}\right)$ are also presented.
\end{abstract}

DOI: 10.1103/PhysRevD.104.054046

\section{INTRODUCTION}

The theory of the strong interaction is $\mathrm{QCD}$, which due to its underlying non-Abelian structure becomes nonperturbative at low energies. Hence, in this region, it is impractical to study the related effects directly within the fundamental theory, and alternative approaches have to be explored. Chiral perturbation theory (ChPT) [1,2] is the effective field theory that can be used to study interactions of hadrons in the low-energy domain. Many observables are known in ChPT to a high loop order, but only recently, it has become of interest to calculate the six-pion amplitude at low energies after it was estimated using lattice QCD, as shortly discussed further below. Therefore, a calculation to next-to-leading order (NLO) in ChPT shall be useful.

The six-pion amplitude at tree level was first done using current algebra methods; see, e.g., Ref. [3]. It has been redone with Lagrangian methods many times; see Ref. [4] and references therein. Until now, it has not been known to one-loop order; the tree-level result at NLO was done before in Refs. [4,5].

Two-flavor ChPT is equivalent to a massive $\mathrm{O}(4) / \mathrm{O}(3)$ nonlinear sigma model extended to higher orders in the expansion. Furthermore, there has been a recent development on the structure of amplitudes, also for scalar theories; see, e.g., Refs. [4-9]. We have therefore calculated at NLO the six-pion amplitude as well as the four-pion amplitude, pion mass, and decay constant for the ChPT generalization to the $\mathrm{O}(N+1) / \mathrm{O}(N)$ massive nonlinear sigma model,

\footnotetext{
johan.bijnens@ @thep.lu.se

tomas.husek@thep.lu.se
}

Published by the American Physical Society under the terms of the Creative Commons Attribution 4.0 International license. Further distribution of this work must maintain attribution to the author(s) and the published article's title, journal citation, and DOI. Funded by SCOAP. which might be useful beyond the QCD two-flavor setting. For three or more flavors, one needs to consider the symmetry breaking pattern $\mathrm{SU}\left(N_{\mathrm{f}}\right) \times \mathrm{SU}\left(N_{\mathrm{f}}\right) / \mathrm{SU}\left(N_{\mathrm{f}}\right)$, with $N_{\mathrm{f}}$ standing for the number of flavors. The six-pion amplitude in this case is a possible direction for future work.

In this paper, we discuss the calculation and the result of the six-pion amplitude. Because of the large number of kinematic invariants in a six-pion amplitude, the reduction to master integrals (i.e., the scalar triangle integrals) leads to an enormous expression. We have chosen a redundant basis of integrals that have good symmetry properties allowing us to write the amplitude in a fairly compact form.

Three-pion systems corresponding to the six-pion amplitude have been extensively studied in lattice QCD [10-17]. The relation of our calculation to the measurement on the lattice is nontrivial to implement, given the complexity of the three-body finite volume calculations and the subtraction of the two-body rescatterings involved. The main original and some later works are Refs. [18-24]. There are also recent reviews introducing the subject and containing many more references $[25,26]$.

In Sec. II, we define the $\mathrm{O}(N+1) / \mathrm{O}(N)$ massive nonlinear sigma model as an extension of two-flavor ChPT. We describe the model, its renormalization at NLO, and the use of more than one parametrization as a check on our results. The four-pion amplitude, pion mass and decay constant are derived in Sec. III. Our main result, the six-pion amplitude, is discussed in Sec. IV, both its calculation and form. Most of the explicit expressions are relegated to Appendix B. Some examples of numerical results are given in Sec. V, and our conclusions are shortly discussed in Sec. VI. Finally, Appendix A discusses the integrals we use.

The analytical work in this manuscript was done both using Wolfram Mathematica with FEYNCALC package [27-29] and a FORM [30] implementation. The numerical results use LoopToOLs [31,32]. 


\section{THEORETICAL SETTING}

The massive $\mathrm{O}(N+1) / \mathrm{O}(N)$ nonlinear sigma model Lagrangian reads

$$
\mathcal{L}_{\mathrm{nL} \sigma \mathrm{M}}=\frac{F^{2}}{2} \partial_{\mu} \Phi^{\top} \partial^{\mu} \Phi+F^{2} \chi^{\top} \Phi
$$

with $\Phi$ being a real vector of $N+1$ components, which transforms as the fundamental representation of $\mathrm{O}(N+1)$ and satisfies $\Phi^{\top} \Phi=1$. The spontaneous symmetry breaking of $\mathrm{O}(N+1)$ to $\mathrm{O}(N)$ is triggered by the vacuum, which takes the form

$$
\left\langle\Phi^{\top}\right\rangle=(1, \overrightarrow{0}) .
$$

The term in Eq. (1) containing $\chi$ explicitly breaks the symmetry to the same $\mathrm{O}(N)$, setting

$$
\chi^{\top}=\left(M^{2}, \overrightarrow{0}\right) .
$$

Above, $F$ and $M$ are the bare pion decay constant and mass, respectively. For $N=3$ meson flavors, the Lagrangian (1) corresponds to the lowest-order Lagrangian of two(-quark)flavor Chiral Perturbation Theory. This can be extended beyond the leading order (LO) in the following way:

$$
\begin{aligned}
\mathcal{L}= & \mathcal{L}_{\mathrm{nL} \sigma \mathrm{M}}+l_{1}\left(\partial_{\mu} \Phi^{\top} \partial^{\mu} \Phi\right)\left(\partial_{\nu} \Phi^{\top} \partial^{\nu} \Phi\right) \\
& +l_{2}\left(\partial_{\mu} \Phi^{\top} \partial_{\nu} \Phi\right)\left(\partial^{\mu} \Phi^{\top} \partial^{\nu} \Phi\right) \\
& +l_{3}\left(\chi^{\top} \Phi\right)^{2}+l_{4} \partial_{\mu} \chi^{\top} \partial^{\mu} \Phi
\end{aligned}
$$

We use the above Lagrangian to calculate the amplitudes in question at the NLO. External fields, as needed for the decay constant, can be added as in Ref. [2]. The coefficients (low-energy constants) $l_{i}$ are free parameters in the theory and carry both UV divergent and finite parts expressed as

$$
l_{i}=(c \mu)^{d-4}\left(\frac{1}{16 \pi^{2}} \frac{1}{d-4} \gamma_{i}+l_{i}^{\mathrm{r}}\right) .
$$

Above, $c$ is such that

$$
\log c=-\frac{1}{2}\left(1-\gamma_{\mathrm{E}}+\log 4 \pi\right) .
$$

Consequently, in terms of $\epsilon=2-d / 2$ and

$$
\frac{1}{\tilde{\epsilon}} \equiv \frac{1}{\epsilon}-\gamma_{\mathrm{E}}+\log 4 \pi-\log \mu^{2}+1,
$$

one writes

$$
l_{i}=-\kappa \frac{\gamma_{i}}{2} \frac{1}{\tilde{\epsilon}}+l_{i}^{\mathrm{r}},
$$

with $\kappa \equiv 1 /\left(16 \pi^{2}\right)$. Notice the extra +1 term in Eq. (7) with respect to the $\overline{\mathrm{MS}}$ renormalization scheme. When considering the four-pion amplitude at the order $\mathcal{O}\left(p^{4}\right)$, the coefficients $\gamma_{i}$ are uniquely fixed by the requirement of the cancellation of the divergent parts among the NLO treelevel (containing vertices with $l_{i} \mathrm{~s}$ ) and one-loop contributions. From studying the pion mass, decay constant, and the four-pion amplitude discussed in the following section, we find

$$
\begin{aligned}
& \gamma_{1}=\frac{N}{2}-\frac{7}{6}, \\
& \gamma_{2}=\frac{2}{3}, \\
& \gamma_{3}=1-\frac{N}{2}, \\
& \gamma_{4}=N-1 .
\end{aligned}
$$

To expand the Lagrangian (4) in terms of the pion fields $\phi_{i}, i=1, \ldots, N$, one needs to use a particular representation for $\Phi$. Using multiple representations simultaneously then serves - together with the cancellation of the UV divergent parts-as the fundamental cross-check pointing to the validity of the final expression, since, of course, one expects to obtain the same (physical) result, irrespective of the parametrization used in the intermediate steps. We used the following five representations:

$$
\begin{gathered}
\Phi_{1}=\left(\sqrt{1-\varphi}, \frac{\boldsymbol{\phi}^{\top}}{F}\right)^{\top}, \\
\Phi_{2}=\frac{1}{\sqrt{1+\varphi}}\left(1, \frac{\boldsymbol{\phi}^{\top}}{F}\right)^{\top}, \\
\Phi_{3}=\left(1-\frac{1}{2} \varphi, \sqrt{1-\frac{1}{4} \varphi \frac{\boldsymbol{\phi}^{\top}}{F}}\right)^{\top}, \\
\Phi_{4}=\left(\cos \sqrt{\varphi}, \frac{1}{\sqrt{\varphi}} \sin \sqrt{\varphi} \frac{\boldsymbol{\phi}^{\top}}{F}\right)^{\top}, \\
\Phi_{5}=\frac{1}{1+\frac{1}{4} \varphi}\left(1-\frac{1}{4} \varphi, \frac{\boldsymbol{\phi}^{\top}}{F}\right)^{\top} .
\end{gathered}
$$

Above, we denote $\varphi \equiv \frac{\phi^{\top} \phi}{F^{2}}$, with $\phi^{\top}=\left(\phi_{1}, \ldots, \phi_{N}\right)$ being a real vector of $N$ components (flavors) transforming linearly under the unbroken part of the $\mathrm{O}(N)$ symmetry group. The parametrization $\Phi_{1}$ is the one used by Gasser and Leutwyler in Ref. [2], $\Phi_{2}$ is a simple variation, $\Phi_{3}$ is such that the term explicitly breaking the symmetry in Eq. (1) only gives mass terms of $\phi_{i}$ s but no vertices, $\Phi_{4}$ represents the result when one follows the general prescription from Ref. [33], and $\Phi_{5}$ is the one originally introduced by Weinberg [34]. Finally, these are just a few examples of the whole class of parametrizations that keep the $\mathrm{O}(N+1)$ symmetry manifest: 


$$
\Phi=\left(\sqrt{1-\varphi f^{2}(\varphi)}, f(\varphi) \frac{\boldsymbol{\phi}^{\top}}{F}\right)^{\top}
$$

Above, $f(x)$ is any analytical function satisfying $f(0)=1$.

\section{FOUR-PION AMPLITUDE}

Let us start with the four-pion amplitude $A_{4 \pi}$, assuming $N$ flavors of pseudoscalar mesons (pions), since this result has (to our knowledge) not been yet presented in the literature. We write the four-pion amplitude exactly in the form as given in Refs. [35,36], generalized to $N \neq 3$. Note that this is somewhat different from the form given in Ref. [2]: Both results are, of course, equivalent to the given order $\mathcal{O}\left(p^{4}\right)$ but lead to different off-shell extrapolations.

In general, for the on-shell amplitude with all the pion incoming 4-momenta $p_{i}$ and flavors $f_{i}, i=1, \ldots, 4$, $\sum_{i} p_{i}=0$, we can write due to invariance under rotation in the isospin space and crossing symmetry,

$$
\begin{aligned}
& A_{4 \pi}\left(p_{1}, f_{1}, p_{2}, f_{2}, p_{3}, f_{3}, f_{4}\right) \\
& =\delta_{f_{1} f_{2}} \delta_{f_{3} f_{4}} A\left(p_{1}, p_{2}, p_{3}\right) \\
& \quad+\delta_{f_{1} f_{3}} \delta_{f_{2} f_{4}} A\left(p_{3}, p_{1}, p_{2}\right) \\
& \quad+\delta_{f_{2} f_{3}} \delta_{f_{1} f_{4}} A\left(p_{2}, p_{3}, p_{1}\right) .
\end{aligned}
$$

In terms of the Mandelstam variables $s=\left(p_{1}+p_{2}\right)^{2}, t=$ $\left(p_{1}+p_{3}\right)^{2}$ and $u=\left(p_{2}+p_{3}\right)^{2}, s+t+u=4 M^{2}$, one has for the subamplitude $A\left(p_{1}, p_{2}, p_{3}\right)=A(s, t, u)$. The latter can be written up to and including $\mathcal{O}\left(p^{4}\right)$, collecting the contributions order by order, as

$$
A(s, t, u)=A^{(2)}(s, t, u)+A^{(4)}(s, t, u) .
$$

The leading-order tree-level $\mathcal{O}\left(p^{2}\right)$ amplitude stems from a single diagram shown in Fig. 1 (schematically $A_{4 \pi}^{(2)}=\left.\mathcal{M}_{\mathrm{LO}}^{(2)}\right|_{\text {on-shell }}$ ), and the related subamplitude (with LO relations $M \rightarrow M_{\pi}$ and $F \rightarrow F_{\pi}$ ) reads

$$
A^{(2)}(s, t, u)=\frac{1}{F_{\pi}^{2}}\left(s-M_{\pi}^{2}\right) .
$$

At the next-to-leading order, one has one-loop diagrams (two topologies of four one-loop diagrams in total) combined with a counterterm, as shown in Fig. 2, together with NLO field renormalization, and mass and decay-constant redefinitions (at the given order) applied to the LO graph. Schematically, this can be written as

$A_{4 \pi}^{(4)}=\mathcal{M}_{1-\text { loop }}+\mathcal{M}_{\mathrm{CT}}+4\left(Z^{1 / 2}-1\right) \mathcal{M}_{\mathrm{LO}}^{(2)}+\mathcal{M}_{\mathrm{LO}}^{(4)}$.

Note that, while the above combination is parametrization independent, the separate terms are not. The matrix element $\mathcal{M}_{1 \text {-loop }}$ is obtained from the diagrams in Figs. 2(a), 2(b), and $\mathcal{M}_{\mathrm{CT}}$ relates to Fig. 2(c). The $Z$ factor used for the field renormalization is related to the pion self-energy $\Sigma$ as

$$
\frac{1}{Z}=1-\left.\frac{\partial \Sigma\left(p^{2}\right)}{\partial p^{2}}\right|_{p^{2}=M_{\pi}^{2}},
$$

with $-i \Sigma$ being represented by a tadpole graph with two external legs plus a counterterm stemming from the $l_{3}$ term in the Lagrangian (4). The matrix element $\mathcal{M}_{\mathrm{LO}}^{(4)}$ denotes the NLO part of the extension of the LO vertex obtained in terms of the replacements

$$
\begin{aligned}
M^{2} & \rightarrow M_{\pi}^{2}+\bar{\Sigma}, \\
\frac{1}{F^{2}} & \rightarrow \frac{1}{F_{\pi}^{2}}(1+2 \delta F)
\end{aligned}
$$

[at the given order equivalent to standard $M_{\pi}^{2}=M^{2}-\bar{\Sigma}$, $\left.F_{\pi}=F(1+\delta F)\right]$, with

$$
\begin{aligned}
\bar{\Sigma} & =\frac{M_{\pi}^{4}}{F_{\pi}^{2}}\left[2 l_{3}^{\mathrm{r}}+\frac{1}{2}(N-2) L\right]+\mathcal{O}\left(\frac{1}{F_{\pi}^{4}}\right), \\
\delta F & =\frac{M_{\pi}^{2}}{F_{\pi}^{2}}\left[l_{4}^{\mathrm{r}}-\frac{1}{2}(N-1) L\right]+\mathcal{O}\left(\frac{1}{F_{\pi}^{4}}\right) .
\end{aligned}
$$

Needless to say, in the final result, one only retains the terms relevant at the order $\mathcal{O}\left(p^{4}\right)$; i.e., terms $\mathcal{O}\left(1 / F_{\pi}^{6}\right)$ are systematically neglected. Thus, in the rest of the expression (19), one simply takes $M \rightarrow M_{\pi}$ and $F \rightarrow F_{\pi}$. Finally, the NLO part of the subamplitude can be written fairly compactly as of the momenta in the following way:

$$
\begin{aligned}
F_{\pi}^{4} A^{(4)}(s, t, u)= & (t-u)^{2}\left(-\frac{5}{36} \kappa-\frac{1}{6} L+\frac{1}{2} l_{2}^{\mathrm{r}}\right)+M_{\pi}^{2} s\left[\left(N-\frac{29}{9}\right) \kappa+\left(N-\frac{11}{3}\right) L-8 l_{1}^{\mathrm{r}}+2 l_{4}^{\mathrm{r}}\right] \\
& +s^{2}\left[\left(\frac{11}{12}-\frac{N}{2}\right) \kappa+\left(1-\frac{N}{2}\right) L+2 l_{1}^{\mathrm{r}}+\frac{1}{2} l_{2}^{\mathrm{r}}\right]+M_{\pi}^{4}\left[\left(\frac{20}{9}-\frac{N}{2}\right) \kappa+\left(\frac{8}{3}-\frac{N}{2}\right) L+8 l_{1}^{\mathrm{r}}+2 l_{3}^{\mathrm{r}}-2 l_{4}^{\mathrm{r}}\right] \\
& +\bar{J}(s)\left[\left(\frac{N}{2}-1\right) s^{2}+(3-N) M_{\pi}^{2} s+\left(\frac{N}{2}-2\right) M_{\pi}^{4}\right]+\left\{\frac{1}{6} \bar{J}(t)\left[2 t^{2}-10 M_{\pi}^{2} t-4 M_{\pi}^{2} s+s t+14 M_{\pi}^{4}\right]\right. \\
& +(t \leftrightarrow u)\} .
\end{aligned}
$$




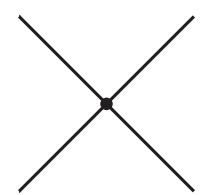

FIG. 1. Leading-order contribution to the four-pion amplitude, with the vertex stemming from $\mathcal{L}_{\mathrm{nL} \sigma \mathrm{M}}$.

Above, we have used

$$
L \equiv \kappa \log \frac{M_{\pi}^{2}}{\mu^{2}} .
$$

The expressions presented in this section agree with the known results for $N=3$ [2], as well as with those on the $N$ dependence (see, e.g., Refs. [37-39]).

\section{SIX-PION AMPLITUDE}

Before we get to discussing the amplitude itself, let us first talk a bit about combinatorics, since in the case of the

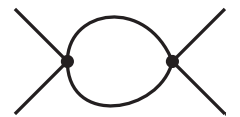

(a) $3 \times$

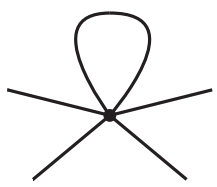

(b) $1 \times$

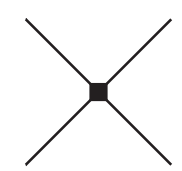

(c) $1 \times$
FIG. 2. NLO topologies relevant for the calculation of the fourpion amplitude. The multiplicities of the respective diagrams are quoted as subcaptions. The standard dotlike vertices come from $\mathcal{L}_{\mathrm{nL} \sigma \mathrm{M}}$, while the square vertex stems from the terms proportional to $l_{i} \mathrm{~s}$ in Eq. (4). As indicated in the main text, there are other diagrams not explicitly depicted here, relevant at NLO for the field renormalization, and mass and decay-constant redefinitions.

six-pion amplitude things get noticeably more complicated compared to the four-pion case where only three different channels/permutations could appear (when distributing four pions in two pairs). There are ten ways one can distribute the six pions in two groups of three. We denote these permutations as $P_{10}$ with

$$
\begin{aligned}
(i, j, k)(l, m, n)=\{ & (1,2,3)(4,5,6),(1,2,4)(3,5,6),(1,2,5)(3,4,6),(1,2,6)(3,4,5),(1,3,4)(2,5,6),(1,3,5)(2,4,6), \\
& (1,3,6)(2,4,5),(1,4,5)(2,3,6),(1,4,6)(2,3,5),(1,5,6)(2,3,4)\} .
\end{aligned}
$$

Similarly, there are 15 ways the six pions can be distributed in three pairs. These we denote as $P_{15}$ and are

$$
\begin{aligned}
(i, j)(k, l)(m, n)=\{ & (1,2)(3,4)(5,6),(1,2)(3,5)(4,6),(1,2)(3,6)(4,5),(1,3)(2,4)(5,6),(1,3)(2,5)(4,6), \\
& (1,3)(2,6)(4,5),(1,4)(2,3)(5,6),(1,4)(2,5)(3,6),(1,4)(2,6)(3,5),(1,5)(2,3)(4,6), \\
& (1,5)(2,4)(3,6),(1,5)(2,6)(3,4),(1,6)(2,3)(4,5),(1,6)(2,4)(3,5),(1,6)(2,5)(3,4)\} .
\end{aligned}
$$

We define a general six-pion amplitude with all the pion incoming 4-momenta $p_{i}$ and flavors $f_{i}, i=1, \ldots, 6$, so it is a function of $p_{1}, f_{1}, \ldots, p_{6}, f_{6}$. The full six-pion amplitude at $\mathcal{O}\left(p^{4}\right)$ can be written as

$$
A_{6 \pi}=A_{6 \pi}^{(4 \pi)}+A_{6 \pi}^{(6 \pi)} .
$$

Above, $A_{6 \pi}^{(4 \pi)}$ is the part that can be written in terms of the four-pion amplitude, and $A_{6 \pi}^{(6 \pi)}$ is the remainder. The first part contains a single pole and is of the form

$$
\begin{aligned}
A_{6 \pi}^{(4 \pi)} \equiv & \sum_{P_{10}, f_{\mathrm{o}}} A_{4 \pi}\left(p_{i}, f_{i}, p_{j}, f_{j}, p_{k}, f_{k}, f_{\mathrm{o}}\right) \\
& \times \frac{(-1)}{p_{i j k}^{2}-M_{\pi}^{2}} A_{4 \pi}\left(p_{l}, f_{l}, p_{m}, f_{m}, p_{n}, f_{n}, f_{\mathrm{o}}\right),
\end{aligned}
$$

with $f_{\mathrm{o}}$ being the flavor of the internal propagator and $p_{i j k} \equiv p_{i}+p_{j}+p_{k}$. Above, $A_{4 \pi}\left(p_{i}, f_{i}, p_{j}, f_{j}, p_{k}, f_{k}, f_{\mathrm{o}}\right)$ is the four-pion amplitude with one leg off shell. Similarly to Eq. (16), we write

$$
\begin{aligned}
A_{4 \pi}\left(p_{i}, f_{i}, p_{j}, f_{j}, p_{k}, f_{k}, f_{\mathrm{o}}\right)= & \delta_{f_{i} f_{j}} \delta_{f_{k} f_{\mathrm{o}}} A\left(p_{i}, p_{j}, p_{k}\right) \\
& +\delta_{f_{i} f_{k}} \delta_{f_{j} f_{\mathrm{o}}} A\left(p_{k}, p_{i}, p_{j}\right) \\
& +\delta_{f_{j} f_{k}} \delta_{f_{i} f_{\mathrm{o}}} A\left(p_{j}, p_{k}, p_{i}\right)
\end{aligned}
$$

The (four-pion) subamplitude $A\left(p_{i}, p_{j}, p_{k}\right)=A(s, t, u)$ is defined as usual, with $s=\left(p_{i}+p_{j}\right)^{2}, t=\left(p_{i}+p_{k}\right)^{2}$ and $u=\left(p_{j}+p_{k}\right)^{2}$. However, note that these variables now satisfy $s+t+u=3 M_{\pi}^{2}+p_{i j k}^{2}$. In Eq. (28), the residue at the pole is unique (related to the uniqueness of the on-shell four-pion amplitude); however, the off-shell extrapolation away from $p_{i j k}^{2}=M_{\pi}^{2}$ is not and is subject to a choice determining how the particular contributions are redistributed among the two parts of Eq. (27). This split into the factorizable $\left(A_{6 \pi}^{(4 \pi)}\right)$ and nonfactorizable $\left(A_{6 \pi}^{(6 \pi)}\right)$ parts is convenient since there are momentum configurations where the intermediate propagators (appearing subsequently only in $\left(A_{6 \pi}^{(4 \pi)}\right)$ can become on shell. At the same time, as discussed later on, this structure naturally shows up 


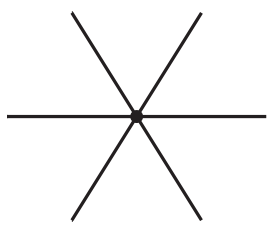

(a) $1 \times$

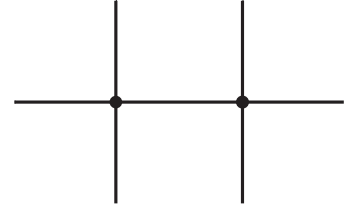

(b) $10 \times$
FIG. 3. Leading-order contributions to the six-pion amplitude. The topology depicted on the right in the subfigure (b) manifests itself with ten contributions with different permutations of the external legs.

diagrammatically at NLO. It is then anticipated that—going beyond the six-particle amplitude-similar comments apply: residues at poles are unique, but away from the poles, there are many more ambiguities.

For the subamplitude $A(s, t, u)$, we choose the form as given by Eqs. (18) and (23). Other off-shell extrapolations are possible and will lead to a different $A_{6 \pi}^{(6 \pi)}$, which has no poles, only cuts, ${ }^{1}$ and can be written in the form

$A_{6 \pi}^{(6 \pi)} \equiv \sum_{P_{15}} \delta_{f_{i} f_{j}} \delta_{f_{k} f_{l}} \delta_{f_{m} f_{n}} A\left(p_{i}, p_{j}, p_{k}, p_{l}, p_{m}, p_{n}\right)$.

The (six-pion) subamplitude $A\left(p_{1}, p_{2}, p_{3}, p_{4}, p_{5}, p_{6}\right)$ should be thought of as a function of three pairs of momenta. It is fully symmetric under the interchange of any of the pairs as well as symmetric for the interchange within a pair. We have chosen a particular form for the offshell four-pion subamplitude $A(s, t, u)$, independent of the parametrization used, so the amplitude $A_{4 \pi}$ and the respective parts $A_{6 \pi}^{(4 \pi)}$ and $A_{6 \pi}^{(6 \pi)}$ from Eq. (27) are, as a consequence, parametrization independent. However, the way the contributions from the one-particle irreducible and reducible diagrams are distributed within the final result is parametrization dependent.

Regarding the six-pion amplitude at the leading order, there are $1+10$ tree diagrams depicted in Fig. 3. The diagram in Fig. 3(a) only contributes to $A_{6 \pi}^{(6 \pi)}$, but the one in Fig. 3(b) contributes to both the pole and nonpole parts $A_{6 \pi}^{(4 \pi)}$ and $A_{6 \pi}^{(6 \pi)}$, respectively. At the next-to-leading order, the one-particle irreducible (1PI) diagrams shown in Figs. 4(a), 4(b), 4(c), and 4(i) clearly contribute only to $A_{6 \pi}^{(6 \pi)}$, together with the field, mass, and decay-constant renormalizations at NLO applied to the LO expression stemming from the first LO graph (in Fig. 3(a)). Schematically,

\footnotetext{
${ }^{1}$ This is not quite true; the imaginary part of the triangle integrals can contain poles.
}

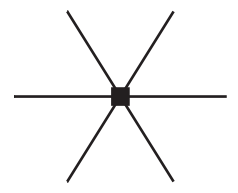

(a) $1 \times$

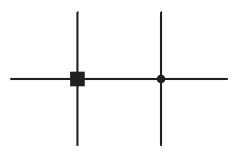

(d) $20 \times$

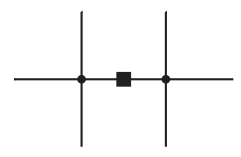

(g) $10 \times$

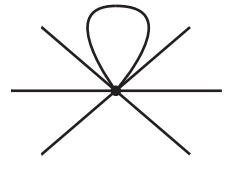

(b) $1 \times$

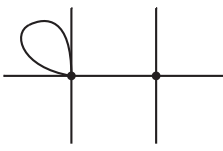

(e) $20 \times$

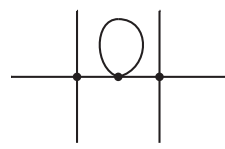

(h) $10 \times$

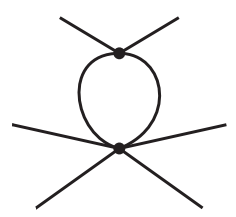

(c) $15 \times$

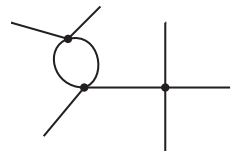

(f) $60 \times$

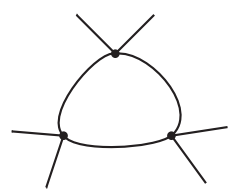

(i) $15 \times$
FIG. 4. NLO topologies relevant for the six-pion amplitude. The multiplicities of the respective diagrams are quoted as subcaptions.

$\left.A_{6 \pi}^{(6 \pi)}\right|_{\mathrm{NLO}, 1 \mathrm{PI}}$

$=\tilde{\mathcal{M}}_{1-\text { loop }}^{(6 \pi)}+\tilde{\mathcal{M}}_{\mathrm{CT}}^{(6 \pi)}+6\left(Z^{1 / 2}-1\right) \tilde{\mathcal{M}}_{\mathrm{LO}}^{(a)(2)}+\tilde{\mathcal{M}}_{\mathrm{LO}}^{(a)(4)}$.

The discussion of the pole part is somewhat more complicated. The double-pole part of the contributions represented by diagrams depicted in Figs. 4(g) and 4(h) cancels with the piece due to the propagator mass renormalization in the LO pole contribution, and consequently

$$
\begin{aligned}
& \tilde{\mathcal{M}}^{2-\text { pole }}+6\left(Z^{1 / 2}-1\right) \tilde{\mathcal{M}}_{\mathrm{LO}}^{(b)(2)}+\left.\tilde{\mathcal{M}}_{\mathrm{LO}}^{(b)(4)}\right|_{\text {prop. }} \\
& =8\left(Z^{1 / 2}-1\right) \tilde{\mathcal{M}}_{\mathrm{LO}}^{(b)(2)} .
\end{aligned}
$$

This, together with $\tilde{\mathcal{M}}_{\mathrm{LO}}^{(b)(2)}+\left.\tilde{\mathcal{M}}_{\mathrm{LO}}^{(b)(4)}\right|_{\text {vert. }}$ and contributions stemming from the topologies shown in Figs. 4(d), 4(e), and 4(f) gives, schematically, the equivalent of two NLO $\pi \pi$ scatterings connected with the propagator, i.e., precisely the structure of Eq. (28). Choosing the particular off-shell form of $A_{6 \pi}^{(4 \pi)}$ as discussed earlier, the remainder is deferred to $A_{6 \pi}^{(6 \pi)}$.

Finally, let us present the results. The six-pion subamplitude can be again written in the following form respecting orders in the expansion we use:

$$
\begin{aligned}
A\left(p_{1}, p_{2}, p_{3}, p_{4}, p_{5}, p_{6}\right)= & A^{(2)}\left(p_{1}, p_{2}, p_{3}, p_{4}, p_{5}, p_{6}\right) \\
& +A^{(4)}\left(p_{1}, p_{2}, p_{3}, p_{4}, p_{5}, p_{6}\right) .
\end{aligned}
$$

At the leading order, we find a simple expression: 


$$
\begin{aligned}
& A^{(2)}\left(p_{1}, p_{2}, p_{3}, p_{4}, p_{5}, p_{6}\right) \\
& =\frac{1}{F_{\pi}^{4}}\left(2 p_{1} \cdot p_{2}+2 p_{3} \cdot p_{4}+2 p_{5} \cdot p_{6}+3 M_{\pi}^{2}\right) \\
& =\frac{1}{F_{\pi}^{4}}\left(q_{1}^{2}+q_{2}^{2}+q_{3}^{2}-3 M_{\pi}^{2}\right) .
\end{aligned}
$$

Above, we used $q_{1}=p_{1}+p_{2}, q_{2}=p_{3}+p_{4}, q_{1}=p_{5}+p_{6}$. Note that the dependence on momenta is the only one at this order compatible with the symmetries of the amplitude. This expression agrees with known results; see, e.g., Refs. [3,4,11].

The main new result presented in this work is the nextorder six-pion subamplitude. We split it up into numerous parts:

$$
\begin{aligned}
F_{\pi}^{6} A^{(4)}\left(p_{1}, p_{2}, \ldots, p_{6}\right)= & A_{C_{3}}+A_{C_{21}}^{(1)}+A_{C_{21}}^{(2)}+A_{C_{11}}+A_{C}^{(1)} \\
& +A_{C}^{(2)}+A_{C}^{(3)}+A_{J}^{(1)}+A_{J}^{(2)} \\
& +A_{\pi}+A_{L}+A_{l} .
\end{aligned}
$$

Each of the terms on the right-hand side has the required symmetries under interchange of momenta. In the above expression, we suppressed the arguments $\left(p_{1}, p_{2}, \ldots, p_{6}\right) \equiv\left(p_{1}, p_{2}, p_{3}, p_{4}, p_{5}, p_{6}\right)$. Not to break the flow of the paper, we moved the results for each of the above parts to Appendix B.

\section{NUMERICAL RESULTS}

We only present a few numerical results here since the full analysis of the finite volume and the subtraction of the two-body rescatterings is very nontrivial; see Refs. [25,26] and references therein.

We choose a symmetric three to three scattering configuration given by

$$
\begin{aligned}
& p_{1}=\left(E_{p}, p, 0,0\right), \\
& p_{2}=\left(E_{p},-\frac{1}{2} p, \frac{\sqrt{3}}{2} p, 0\right), \\
& p_{3}=\left(E_{p},-\frac{1}{2} p,-\frac{\sqrt{3}}{2} p, 0\right), \\
& p_{4}=\left(-E_{p}, 0,0, p\right), \\
& p_{5}=\left(-E_{p}, \frac{\sqrt{3}}{2} p, 0,-\frac{1}{2} p\right), \\
& p_{6}=\left(-E_{p},-\frac{\sqrt{3}}{2} p, 0,-\frac{1}{2} p\right),
\end{aligned}
$$

with $E_{p}=\sqrt{p^{2}+M^{2}}$. The numerical inputs we use are

$$
\begin{aligned}
M_{\pi} & =0.139570 \mathrm{GeV}, & & \bar{l}_{1}=-0.4, \\
F_{\pi} & =0.0927 \mathrm{GeV}, & & \bar{l}_{2}=4.3, \\
\mu & =0.77 \mathrm{GeV}, & & \bar{l}_{3}=3.41, \\
N & =3, & & \bar{l}_{4}=4.51,
\end{aligned}
$$

where the values for $\bar{l}_{i}$ are from Refs. [40-42]. One then obtains $l_{i}^{\mathrm{r}}$ [introduced in Eqs. (5) and (8)] appearing in our results after employing $l_{i}^{r}=\frac{1}{2} \kappa \gamma_{i}\left(\bar{l}_{i}+\ln \left(M_{\pi}^{2} / \mu^{2}\right)\right)$, as defined in Ref. [2].

The six-pion subamplitude as introduced in Eq. (33) is plotted in Fig. 5. As one can see, the corrections are sizable but not enormous. They are, however, much smaller than the part coming from the four-pion amplitude as given in Eq. (28). To compare the same flavor structures, we combine Eqs. (28) and (29) and rewrite the result in the form of Eq. (30). The size of the different contributions for $p=0.1 \mathrm{GeV}$ (the end point of the shown curves) is given in Table I. Even though-at least in this particular kinematical setting - the individual contributions are relatively sizable, cancellations take place. As a result, the overall

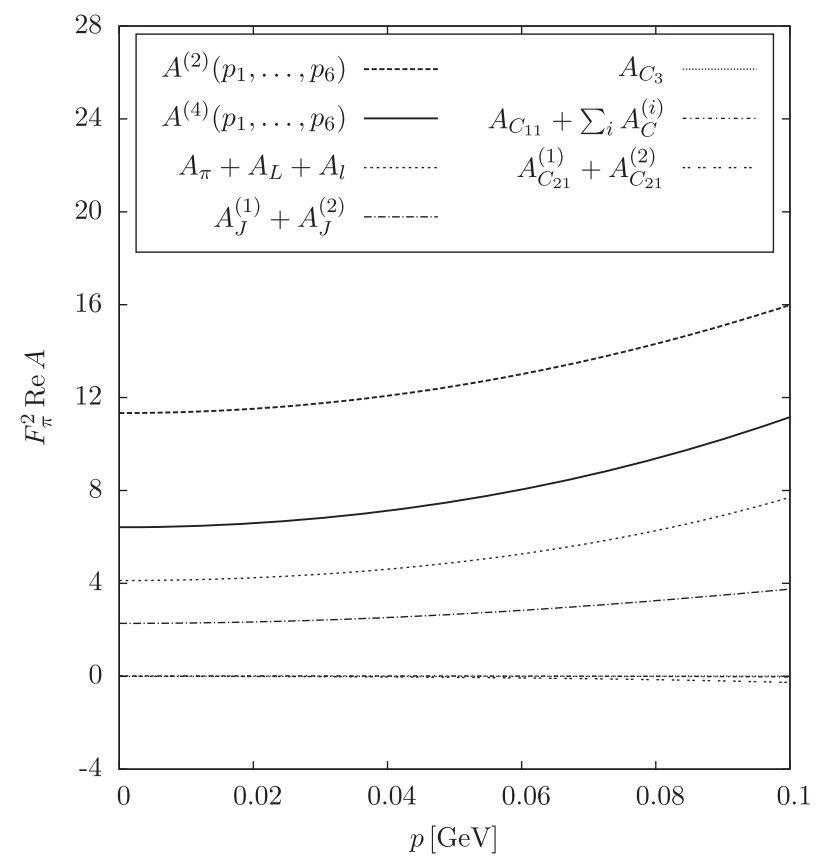

FIG. 5. The six-pion subamplitude in the kinematic configuration of Eq. (36). Plotted are the lowest-order result $A^{(2)}\left(p_{1}, p_{2}, \ldots, p_{6}\right)$ of Eq. (34) and the next-to-leading-order result $A^{(4)}\left(p_{1}, p_{2}, \ldots, p_{6}\right)$ of Eq. (35) together with several groups of its individual constituents. We show only the real parts. To enhance readability, the order in the key is consistent with how the curves appear in the plot. Following Eq. (35), the solid curve representing the NLO result is the sum of all the curves below it. The lines corresponding to $A_{C_{3}}$ and $A_{C_{11}}+$ $\sum_{i} A_{C}^{(i)}$ are added for completeness; in this plot, they lie very close to zero and basically overlap. 
TABLE I. The different contributions of the four-pion and sixpion amplitudes [all taken in the flavor-stripped form analogous to Eq. (30)]. LO indicates the lowest order and NLO next-toleading order. The inputs are as given in the main text with $p=0.1 \mathrm{GeV}$. We quote only the real parts. Following Eq. (35), $A^{(4)}\left(p_{1}, p_{2}, \ldots, p_{6}\right)$ is the sum of terms in the bottom part of the table. The amplitudes are multiplied by a fitting power of $F_{\pi}$ consistently with the respective definitions [see, in particular, Eq. (35)] and (notably by $F_{\pi}^{2}$ ) to obtain dimensionless results.

\begin{tabular}{lrrr}
\hline \hline \multicolumn{4}{c}{$F_{\pi}^{2} \operatorname{Re} A$} \\
\hline$A_{6 \pi}^{(4 \pi)}(\mathrm{LO})$ & -319.00 & $A^{(2)}\left(p_{1}, \ldots, p_{6}\right)$ & 15.99 \\
$A_{6 \pi}^{(4 \pi)}(\mathrm{NLO})$ & -28.54 & $A^{(4)}\left(p_{1}, \ldots, p_{6}\right)$ & 11.16 \\
\hline \hline
\end{tabular}

\begin{tabular}{lrrr}
\hline \hline \multicolumn{4}{c}{$F_{\pi}^{2} \times \operatorname{Re} A / F_{\pi}^{6}$} \\
\hline$A_{C_{3}}$ & 0.002 & $A_{J}^{(1)}$ & 1.917 \\
$A_{C_{21}}^{(1)}$ & -0.948 & $A_{J}^{(2)}$ & 1.835 \\
$A_{C_{21}}^{(2)}$ & 0.682 & $A_{\pi}$ & -2.488 \\
$A_{C_{11}}^{(1)}$ & 0.090 & $A_{L}$ & 8.985 \\
$A_{C}^{(1)}$ & -0.026 & $A_{l}$ & 1.209 \\
$A_{C}^{(2)}$ & 0.890 & & \\
$A_{C}^{(3)}$ & -0.984 & & \\
\hline \hline
\end{tabular}

contribution of the triangle functions is negligible compared to the $\bar{J}$ and polynomial parts, which clearly dominate in size also in terms of individual contributions. This can be seen in Table I comparing the left- and righthand sides of the bottom part of the table.

In the limit $p \rightarrow 0$, we find the following analytical expressions,

$$
\left.F_{\pi}^{2} A^{(2)}\left(p_{1}, p_{2}, \ldots, p_{6}\right)\right|_{p \rightarrow 0}=5 \frac{M_{\pi}^{2}}{F_{\pi}^{2}},
$$

$$
\begin{aligned}
F_{\pi}^{2} & \left.\operatorname{Re} A^{(4)}\left(p_{1}, p_{2}, \ldots, p_{6}\right)\right|_{p \rightarrow 0} \\
= & \frac{M_{\pi}^{4}}{F_{\pi}^{4}}\left\{(-44+30 N) \kappa+(24) \kappa-\frac{1}{18}(2+225 N) \kappa\right. \\
& -\frac{1}{6}(14+75 N) L+\left(16 l_{1}^{\mathrm{r}}+56 l_{2}^{\mathrm{r}}+6 l_{3}^{\mathrm{r}}+20 l_{4}^{\mathrm{r}}\right) \\
& \left.+\frac{1}{2} \kappa[-(30-9 N)+(20)-(16)]\right\} .
\end{aligned}
$$

Numerically, $\left.\quad F_{\pi}^{2} A^{(2)}\left(p_{1}, p_{2} \ldots, p_{6}\right)\right|_{p \rightarrow 0} \approx 11.33, \quad$ and $\left.F_{\pi}^{2} \operatorname{Re} A^{(4)}\left(p_{1}, p_{2} \ldots, p_{6}\right)\right|_{p \rightarrow 0} \approx 6.416$. In the case of the NLO amplitude, the numerically dominant part stemming from $A_{J}^{(1)}, A_{J}^{(2)}, A_{\pi}, A_{L}$, and $A_{l}$ already yields 6.400 , and in this exact order, the listed contributions appear in the above expression, the last row of Eq. (39) then being dedicated to the contributions of $A_{C}^{(1)}, A_{C}^{(2)}$, and $A_{C}^{(3)}$.

\section{CONCLUSIONS}

In this paper, we calculated the pion mass, decay constant, and the four-pion and six-pion amplitude to NLO in the massive $\mathrm{O}(N)$ nonlinear sigma model. In Sec. II, we described the model and constructed the relevant NLO Lagrangian in analogy with the two(-quark)-flavor ChPT Lagrangian [2]. The mass, decay constant, and the four-pion amplitude agree for $N=3$ with Ref. [2] and with general- $N$ results of Refs. [37-39].

Our main result is the six-pion amplitude. We split it in one-particle reducible and irreducible parts; see Eq. (27). The reducible part is given in Eq. (28), where we chose to employ the off-shell four-pion amplitude generalizing (beyond $N=3$ ) the amplitude given in Refs. [35,36]. The irreducible part can be divided in a large number of subparts, each satisfying the expected permutation symmetries, as given in Eq. (35), and the expressions are given explicitly in Appendix B. The choice of triangle loop integrals with high symmetry allows for a fairly compact expression.

Some numerical results for one particular momentum configuration are presented in Sec. V. The NLO correction is sizable but not very large.

Work is in progress to combine our results with the methods for extracting three-body scattering from finite volume in lattice QCD. We expect that our result might also be of interest for the amplitude community.

\section{ACKNOWLEDGMENTS}

This work is supported in part by the Swedish Research Council Grants No. 2016-05996 and No. 2019-03779.

\section{APPENDIX A: CONVENTIONS FOR THE LOOP INTEGRALS}

Throughout the paper, we treat the momenta $\left(p_{1}, \ldots, p_{6}\right)$ as incoming. We introduce the following combinations:

$$
\begin{aligned}
& q_{1}=p_{1}+p_{2}, \quad q_{2}=p_{3}+p_{4}, \quad q_{1}=p_{5}+p_{6}, \\
& r_{1}=p_{1}-p_{2}, \quad r_{2}=p_{3}-p_{4}, \quad r_{3}=p_{5}-p_{6} \text {. }
\end{aligned}
$$

The functions we use to represent our results are the standard Passarino-Veltman one-loop integrals. We specify some of them for completeness and to fix our notation. The simpler integrals with one and two propagators read

$$
\begin{aligned}
A_{0} & =\frac{1}{i} \int \frac{\mathrm{d}^{d} r}{(2 \pi)^{d}} \frac{1}{r^{2}-M^{2}}=M^{2} \kappa \frac{1}{\tilde{\epsilon}}-M^{2} L, \\
B_{0}\left(q^{2}\right) & =\frac{1}{i} \int \frac{\mathrm{d}^{d} r}{(2 \pi)^{d}} \frac{1}{\left(r^{2}-M^{2}\right)\left[(r-q)^{2}-M^{2}\right]} \\
& =\kappa \frac{1}{\tilde{\epsilon}}-\kappa-L+\bar{J}\left(q^{2}\right),
\end{aligned}
$$


with [as in Eq. (7)]

$$
\frac{1}{\tilde{\epsilon}} \equiv \frac{1}{\epsilon}-\gamma_{\mathrm{E}}+\log 4 \pi-\log \mu^{2}+1 .
$$

We also remind the reader that we set

$$
\kappa=\frac{1}{16 \pi^{2}}, \quad L=\kappa \log \frac{M^{2}}{\mu^{2}} .
$$

In what follows, we use for the Feynman denominators the compact notation

$$
D\left( \pm q_{i}\right) \equiv\left(r \mp q_{i}\right)^{2}-M^{2}
$$

while setting $D_{0} \equiv D(0)=r^{2}-M^{2}$. Then, the integrals $A_{0}$ and $B_{0}$ from Eq. (A2) can be simply written as

$$
\begin{aligned}
A_{0} & =\frac{1}{i} \int \frac{\mathrm{d}^{d} r}{(2 \pi)^{d}} \frac{1}{D_{0}}, \\
B_{0}\left(q_{1}^{2}\right) & =\frac{1}{i} \int \frac{\mathrm{d}^{d} r}{(2 \pi)^{d}} \frac{1}{D_{0} D\left(q_{1}\right)} .
\end{aligned}
$$

It is the tensor triangle one-loop integrals of higher ranks which generate lengthy expressions upon reduction to the scalar ones. Regarding the rank-3 integrals, the following combination has more symmetries than the first term only:

$$
C_{3}\left(p_{1}, p_{2}, \ldots, p_{6}\right)=\frac{1}{3} \frac{1}{i} \int \frac{\mathrm{d}^{d} r}{(2 \pi)^{d}} \frac{r \cdot r_{1} r \cdot r_{2} r \cdot r_{3}}{D_{0}}\left[\frac{1}{D\left(q_{1}\right) D\left(-q_{2}\right)}+\frac{1}{D\left(q_{2}\right) D\left(-q_{3}\right)}+\frac{1}{D\left(q_{3}\right) D\left(-q_{1}\right)}\right] .
$$

Moreover, note that, contrary to a naive (loop-momenta-) power counting applied to separate terms in Eq. (A7), the combination $C_{3}$ is UV finite. One should think of $C_{3}$ as a function of three pairs $\left(p_{1}, p_{2}\right),\left(p_{3}, p_{4}\right)$, and $\left(p_{5}, p_{6}\right)$. Such a combination differs from the first term alone only by terms with two or fewer $r \cdot r_{i}$. One can see this in terms of a shift of the integration variable: One obtains the first propagator from the second one using $r \rightarrow r+q_{2}$ and from the last one using $r \rightarrow r-q_{1}$. The combination $C_{3}\left(p_{1}, p_{2}, p_{3}, p_{4}, p_{5}, p_{6}\right)$ is antisymmetric under the following set of operations (taking each line separately):

$$
\begin{gathered}
p_{1} \leftrightarrow p_{2} ; \\
\left(p_{1}, p_{2}\right) \leftrightarrow\left(p_{3}, p_{4}\right), \quad\left(p_{1}, p_{2}\right) \leftrightarrow\left(p_{5}, p_{6}\right) .
\end{gathered}
$$

Hence, it is antisymmetric under the interchange of the momenta inside each pair and antisymmetric under the interchange of two pairs. Note that this generalization already follows from Eqs. (A8) and (A9).

One can also define symmetric combinations with fewer terms in the numerator (for rank-2 integrals):

$$
\begin{aligned}
& C_{21}\left(p_{1}, p_{2}, \ldots, p_{6}\right)=\frac{1}{i} \int \frac{\mathrm{d}^{d} r}{(2 \pi)^{d}} \frac{r \cdot r_{1} r \cdot r_{2}}{D_{0} D\left(q_{1}\right) D\left(-q_{2}\right)}, \\
& C_{22}\left(p_{1}, p_{2}, \ldots, p_{6}\right)=\frac{1}{i} \int \frac{\mathrm{d}^{d} r}{(2 \pi)^{d}} \frac{r \cdot r_{2} r \cdot r_{3}}{D_{0} D\left(q_{2}\right) D\left(-q_{3}\right)}, \\
& C_{23}\left(p_{1}, p_{2}, \ldots, p_{6}\right)=\frac{1}{i} \int \frac{\mathrm{d}^{d} r}{(2 \pi)^{d}} \frac{r \cdot r_{3} r \cdot r_{1}}{D_{0} D\left(q_{3}\right) D\left(-q_{1}\right)} .
\end{aligned}
$$

Notice the cyclic symmetry. To rewrite the results stemming directly from the diagrams in terms of the integrals $C_{2 i}$, one needs to use the change of integration variables $r \rightarrow r+q_{2}$ and $r \rightarrow r-q_{1}$. The last two integrals $\left(C_{22}\right.$ and $\left.C_{23}\right)$ can be related to the first one $\left(C_{21}\right)$ with pairs interchanged and pieces with at most one $r \cdot r_{i}$. Thus, we only need $C_{21}$ to express the final result. Finally, $C_{21}$ is antisymmetric under the interchange $p_{1} \leftrightarrow p_{2}$ and symmetric under $\left(p_{1}, p_{2}\right) \leftrightarrow\left(p_{3}, p_{4}\right)$ and $p_{5} \leftrightarrow p_{6}$.

The integrals with one product $r \cdot r_{i}$ in the numerator can also be defined in a way symmetric under cyclic interchange:

$$
\begin{aligned}
C_{11}\left(p_{1}, p_{2}, \ldots, p_{6}\right) & =\frac{1}{i} \int \frac{\mathrm{d}^{d} r}{(2 \pi)^{d}} \frac{r \cdot r_{3}}{D_{0} D\left(q_{1}\right) D\left(-q_{2}\right)}, \\
C_{12}\left(p_{1}, p_{2}, \ldots, p_{6}\right) & =\frac{1}{i} \int \frac{\mathrm{d}^{d} r}{(2 \pi)^{d}} \frac{r \cdot r_{1}}{D_{0} D\left(q_{2}\right) D\left(-q_{3}\right)}, \\
C_{13}\left(p_{1}, p_{2}, \ldots, p_{6}\right) & =\frac{1}{i} \int \frac{\mathrm{d}^{d} r}{(2 \pi)^{d}} \frac{r \cdot r_{2}}{D_{0} D\left(q_{3}\right) D\left(-q_{1}\right)} .
\end{aligned}
$$

Using the change of integration variables $r \rightarrow r+q_{2}$ and $r \rightarrow r-q_{1}$, the last two integrals can be again related to the first one with pairs interchanged and parts without $r \cdot r_{i}$ in the numerator. Thus, we only need $C_{11}$ to express the final result. The integral $C_{11}$ is antisymmetric under the interchange $p_{5} \leftrightarrow p_{6},\left(p_{1}, p_{2}\right) \leftrightarrow\left(p_{3}, p_{4}\right)$ and is symmetric under $p_{1} \leftrightarrow p_{2}$ and $p_{3} \leftrightarrow p_{4}$. 
Finally, we define

$C\left(p_{1}, p_{2}, \ldots, p_{6}\right)=\frac{1}{i} \int \frac{\mathrm{d}^{d} r}{(2 \pi)^{d}} \frac{1}{D_{0} D\left(q_{1}\right) D\left(-q_{2}\right)}$,

which is symmetric under $p_{1} \leftrightarrow p_{2}$ and under all pair interchanges.

We express the amplitude in terms of $C_{3}, C_{21}, C_{11}$, and $C$. As already mentioned, the former three can be expressed in terms of $C$, but the expressions are cumbersome and lead to a very long expression for the amplitude. We have therefore kept all these four, among which only $C_{21}$ has a UV infinite part:

$$
\begin{aligned}
& C_{21}\left(p_{1}, p_{2}, p_{3}, p_{4}, p_{5}, p_{6}\right) \\
& \quad=\kappa \frac{r_{1} \cdot r_{2}}{4} \frac{1}{\tilde{\epsilon}}+\bar{C}_{21}\left(p_{1}, p_{2}, p_{3}, p_{4}, p_{5}, p_{6}\right) .
\end{aligned}
$$

A basis of momenta (up to the relation that is only valid in four dimensions) which makes it easier to see symmetries is

$$
\begin{gathered}
q_{1}^{2}, q_{2}^{2}, q_{3}^{2}, r_{1} \cdot r_{2}, r_{2} \cdot r_{3}, r_{3} \cdot r_{1}, \\
\left(q_{1}-q_{2}\right) \cdot r_{3}=2 q_{1} \cdot r_{3}, \\
\left(q_{2}-q_{3}\right) \cdot r_{1}=2 q_{2} \cdot r_{1}, \\
\left(q_{3}-q_{1}\right) \cdot r_{2}=2 q_{3} \cdot r_{2} .
\end{gathered}
$$

\section{APPENDIX B: THE SIX-PION AMPLITUDE EXPRESSIONS}

In this section, when we refer to a pair, we mean $\left(p_{1}, p_{2}\right),\left(p_{3}, p_{4}\right)$, or $\left(p_{5}, p_{6}\right)$. Note that in order to get the amplitude in this simpler form we had to use symmetry properties of the integrals as well as many kinematic relations. The notation $R_{i j k l m n}$ indicates that in that term which it multiplies $\left\{p_{1}, p_{2}, p_{3}, p_{4}, p_{5}, p_{6}\right\}$ needs to be replaced by $\left\{p_{i}, p_{j}, p_{k}, p_{l}, p_{m}, p_{n}\right\}$.

Because of the symmetries, only one combination of $C_{3}$ with different arguments can emerge:

$A_{C_{3}}=\left(R_{152346}+R_{152436}+R_{162345}+R_{162435}-R_{132546}-R_{132645}-R_{142536}-R_{142635}\right) C_{3}\left(p_{1}, p_{2}, p_{3}, p_{4}, p_{5}, p_{6}\right)$.

For $\bar{C}_{21}$, two different cases appear. One is when the last two of its arguments (after the replacement operators $R$ are applied) correspond to one of the pairs:

$A_{C_{21}}^{(1)}=\left(R_{132456}+R_{142356}+R_{152634}+R_{162534}+R_{354612}+R_{364512}\right) \bar{C}_{21}\left(p_{1}, p_{2}, p_{3}, p_{4}, p_{5}, p_{6}\right)\left(4 p_{5} \cdot p_{6}+2 M_{\pi}^{2}\right)$.

The other combination is when none of the pairs shows up:

$$
\begin{aligned}
A_{C_{21}}^{(2)}= & \left(R_{132546}+R_{132645}+R_{142536}+R_{142635}+R_{231546}+R_{241536}+R_{231645}+R_{241635}\right. \\
& -R_{234516}-R_{234615}-R_{243516}-R_{243615}+R_{253614}+R_{254613}+R_{263514}+R_{264513} \\
& \left.-R_{143526}+R_{163524}-R_{143625}+R_{153624}-R_{134526}+R_{164523}-R_{134625}+R_{154623}\right) \\
& \times \bar{C}_{21}\left(p_{1}, p_{2}, p_{3}, p_{4}, p_{5}, p_{6}\right)\left(\frac{1}{3}\left(p_{1}+p_{2}\right) \cdot\left(p_{5}-p_{6}\right)-p_{5} \cdot p_{6}\right) .
\end{aligned}
$$

The terms with $C_{11}$ can be written as one combination:

$$
\begin{aligned}
A_{C_{11}}= & \left(R_{132546}+R_{132645}+R_{142536}+R_{142635}-R_{152346}-R_{152436}-R_{162345}-R_{162435}\right. \\
& +R_{234516}+R_{234615}+R_{243516}+R_{243615}+R_{253614}+R_{254613}+R_{263514}+R_{264513} \\
& \left.-R_{351426}-R_{351624}-R_{361425}-R_{361524}-R_{451326}-R_{451623}-R_{461325}-R_{461523}\right) \\
& \times C_{11}\left(p_{1}, p_{2}, p_{3}, p_{4}, p_{5}, p_{6}\right)\left[\left(p_{1} \cdot p_{2}\right)\left(p_{3} \cdot p_{4}\right)\right] .
\end{aligned}
$$

The terms containing $C$ are of three types: with three, one, or none of the pairs. The part with three pairs is

$$
\begin{aligned}
A_{C}^{(1)}= & C\left(p_{1}, p_{2}, p_{3}, p_{4}, p_{5}, p_{6}\right)\left[-N M_{\pi}^{6}-2(N-1) M_{\pi}^{4}\left(p_{1} \cdot p_{2}+p_{3} \cdot p_{4}+p_{5} \cdot p_{6}\right)\right. \\
& \left.-4(N-2) M_{\pi}^{2}\left(p_{1} \cdot p_{2} p_{3} \cdot p_{4}+p_{3} \cdot p_{4} p_{5} \cdot p_{6}+p_{5} \cdot p_{6} p_{1} \cdot p_{2}\right)-8(N-3) p_{1} \cdot p_{2} p_{3} \cdot p_{4} p_{5} \cdot p_{6}\right] .
\end{aligned}
$$

Those with one pair are 


$$
\begin{aligned}
A_{C}^{(2)}= & \left(R_{123546}+R_{123645}+R_{341526}+R_{341625}+R_{561324}+R_{561423}\right) \\
& \times C\left(p_{1}, p_{2}, p_{3}, p_{4}, p_{5}, p_{6}\right)\left(-4 p_{1} \cdot p_{2}-2 M_{\pi}^{2}\right) p_{3} \cdot p_{4} p_{5} \cdot p_{6} .
\end{aligned}
$$

The case when no pair is present in the arguments of $C$ s reads

$$
\begin{aligned}
A_{C}^{(3)}= & \left(R_{132546}+R_{132645}+R_{142536}+R_{142635}+R_{152364}+R_{152463}+R_{162354}+R_{162453}\right) \\
& \times C\left(p_{1}, p_{2}, p_{3}, p_{4}, p_{5}, p_{6}\right)\left[p_{1} \cdot p_{2} p_{3} \cdot p_{4} p_{5} \cdot p_{6}-p_{1} \cdot p_{2} p_{3} \cdot p_{4}\left(p_{1}+p_{2}\right) \cdot\left(p_{5}-p_{6}\right)\right. \\
& \left.-p_{3} \cdot p_{4} p_{5} \cdot p_{6}\left(p_{3}+p_{4}\right) \cdot\left(p_{1}-p_{2}\right)+p_{5} \cdot p_{6} p_{1} \cdot p_{2}\left(p_{5}+p_{6}\right) \cdot\left(p_{3}-p_{4}\right)\right] .
\end{aligned}
$$

The terms containing $\bar{J}$ are similarly split in two expressions depending on whether the argument of $\bar{J}$ corresponds to a pair or not:

$$
\begin{aligned}
A_{J}^{(1)}= & \left(1+R_{341256}+R_{561234}\right) \bar{J}\left(\left(p_{1}+p_{2}\right)^{2}\right)\left[(2 N-3)\left(p_{1} \cdot p_{2}\right)^{2}+(2 N-7) p_{1} \cdot p_{2}\left(p_{3} \cdot p_{4}+p_{5} \cdot p_{6}\right)\right. \\
& \left.+\left(4 N-\frac{13}{2}\right) M_{\pi}^{2} p_{1} \cdot p_{2}+\left(N-\frac{5}{2}\right) M_{\pi}^{2}\left(p_{3} \cdot p_{4}+p_{5} \cdot p_{6}\right)+\frac{3}{2}(N-1) M_{\pi}^{4}\right] \\
A_{J}^{(2)}= & \left(R_{132456}+R_{142356}+R_{231456}+R_{241356}+R_{152634}+R_{251634}\right. \\
& \left.+R_{162543}+R_{261543}+R_{536412}+R_{635421}+R_{546312}+R_{645321}\right) \\
& \times \bar{J}\left(\left(p_{1}+p_{2}\right)^{2}\right)\left\{\frac{3}{4} p_{5} \cdot p_{6}\left(p_{5} \cdot p_{6}-p_{1} \cdot p_{3}-p_{2} \cdot p_{4}\right)-\frac{3}{8} M_{\pi}^{2}\left(p_{1} \cdot p_{3}+p_{2} \cdot p_{4}+3 p_{5} \cdot p_{6}\right)\right. \\
& +\frac{1}{24}\left(p_{1}-p_{3}\right) \cdot\left(p_{2}-p_{4}\right)\left[2\left(p_{1} \cdot p_{2}-p_{3} \cdot p_{4}\right)+19 p_{5} \cdot p_{6}-p_{1} \cdot p_{3}-p_{2} \cdot p_{4}+4 M_{\pi}^{2}\right] \\
& \left.+\frac{1}{24}\left[\left(p_{1}-p_{3}\right) \cdot\left(p_{2}-p_{4}\right)\right]^{2}+\frac{1}{2}\left(p_{1} \cdot p_{2}-p_{3} \cdot p_{4}\right)\left(3 p_{5} \cdot p_{6}+M_{\pi}^{2}\right)-\frac{5}{8} M_{\pi}^{4}\right\} .
\end{aligned}
$$

The polynomial part is finally

$$
\begin{gathered}
A_{\pi}=\kappa\left[\left(\frac{49}{144}-\frac{N}{2}\right)\left(q_{1}^{4}+q_{2}^{4}+q_{3}^{4}\right)+\left(\frac{281}{72}-N\right)\left(q_{1}^{2} q_{2}^{2}+q_{2}^{2} q_{3}^{2}+q_{3}^{2} q_{1}^{2}\right)-\left(\frac{217}{18}-3 N\right) M_{\pi}^{2}\left(q_{1}^{2}+q_{2}^{2}+q_{3}^{2}\right)\right. \\
\left.-\frac{5}{36}\left[\left(r_{1} \cdot r_{2}\right)^{2}+\left(r_{2} \cdot r_{3}\right)^{2}+\left(r_{3} \cdot r_{1}\right)^{2}\right]+\left(23-\frac{9}{2} N\right) M_{\pi}^{4}\right], \\
A_{L}=L\left[\left(\frac{1}{3}-\frac{N}{2}\right)\left(q_{1}^{4}+q_{2}^{4}+q_{3}^{4}\right)+\left(\frac{13}{3}-N\right)\left(q_{1}^{2} q_{2}^{2}+q_{2}^{2} q_{3}^{2}+q_{3}^{2} q_{1}^{2}\right)-\left(\frac{41}{3}-3 N\right) M_{\pi}^{2}\left(q_{1}^{2}+q_{2}^{2}+q_{3}^{2}\right)\right. \\
\left.-\frac{1}{6}\left[\left(r_{1} \cdot r_{2}\right)^{2}+\left(r_{2} \cdot r_{3}\right)^{2}+\left(r_{3} \cdot r_{1}\right)^{2}\right]+\left(27-\frac{9}{2} N\right) M_{\pi}^{4}\right], \\
A_{l}=\frac{1}{2}\left(4 l_{1}^{\mathrm{r}}+l_{2}^{\mathrm{r}}\right)\left(q_{1}^{2}+q_{2}^{2}+q_{3}^{2}\right)^{2}-\left(20 l_{1}^{\mathrm{r}}+l_{2}^{\mathrm{r}}-4 l_{4}^{\mathrm{r}}\right) M_{\pi}^{2}\left(q_{1}^{2}+q_{2}^{2}+q_{3}^{2}\right) \\
+2 l_{2}^{\mathrm{r}}\left[\left(q_{1} \cdot r_{3}\right)^{2}+\left(q_{2} \cdot r_{1}\right)^{2}+\left(q_{3} \cdot r_{2}\right)^{2}\right]+6\left(8 l_{1}^{\mathrm{r}}+l_{3}^{\mathrm{r}}-2 l_{4}^{\mathrm{r}}\right) M_{\pi}^{4} .
\end{gathered}
$$


[1] S. Weinberg, Phenomenological Lagrangians, Physica (Amsterdam) 96A, 327 (1979).

[2] J. Gasser and H. Leutwyler, Chiral perturbation theory to one loop, Ann. Phys. (N.Y.) 158, 142 (1984).

[3] H. Osborn, Implications of Adler zeros for multipion processes, Lett. Nuovo Cimento 2, 717 (1969).

[4] J. Bijnens, K. Kampf, and M. Sjö, Higher-order tree-level amplitudes in the nonlinear sigma model, J. High Energy Phys. 11 (2019) 074.

[5] I. Low and Z. Yin, Soft bootstrap and effective field theories, J. High Energy Phys. 11 (2019) 078.

[6] C. Cheung, K. Kampf, J. Novotný, C.-H. Shen, and J. Trnka, On-Shell Recursion Relations for Effective Field Theories, Phys. Rev. Lett. 116, 041601 (2016).

[7] C. Cheung, K. Kampf, J. Novotný, C.-H. Shen, and J. Trnka, A periodic table of effective field theories, J. High Energy Phys. 02 (2017) 020.

[8] K. Kampf, J. Novotný, M. Shifman, and J. Trnka, New Soft Theorems for Goldstone Boson Amplitudes, Phys. Rev. Lett. 124, 111601 (2020).

[9] I. Low, L. Rodina, and Z. Yin, Double copy in higher derivative operators of Nambu-Goldstone bosons, Phys. Rev. D 103, 025004 (2021).

[10] M. Mai and M. Doring, Finite-Volume Spectrum of $\pi^{+} \pi^{+}$ and $\pi^{+} \pi^{+} \pi^{+}$Systems, Phys. Rev. Lett. 122, 062503 (2019).

[11] T. D. Blanton, F. Romero-López, and S. R. Sharpe, $I=3$ Three-Pion Scattering Amplitude from Lattice QCD, Phys. Rev. Lett. 124, 032001 (2020).

[12] M. Mai, M. Döring, C. Culver, and A. Alexandru, Threebody unitarity versus finite-volume $\pi^{+} \pi^{+} \pi^{+}$spectrum from lattice QCD, Phys. Rev. D 101, 054510 (2020).

[13] C. Culver, M. Mai, R. Brett, A. Alexandru, and M. Döring, Three pion spectrum in the $I=3$ channel from lattice QCD, Phys. Rev. D 101, 114507 (2020).

[14] M. Fischer, B. Kostrzewa, L. Liu, F. Romero-López, M. Ueding, and C. Urbach, Scattering of two and three physical pions at maximal isospin from lattice QCD, Eur. Phys. J. C 81, 436 (2021).

[15] M. T. Hansen, R. A. Briceño, R. G. Edwards, C. E. Thomas, and D. J. Wilson (Hadron Spectrum Collaboration), EnergyDependent $\pi^{+} \pi^{+} \pi^{+}$Scattering Amplitude from QCD, Phys. Rev. Lett. 126, 012001 (2021).

[16] R. Brett, C. Culver, M. Mai, A. Alexandru, M. Döring, and F. X. Lee, Three-body interactions from the finite-volume QCD spectrum, Phys. Rev. D 104, 014501 (2021).

[17] T. D. Blanton, A. D. Hanlon, B. Hörz, C. Morningstar, F. Romero-López, and S. R. Sharpe, Interactions of two and three mesons including higher partial waves from lattice QCD, arXiv:2106.05590.

[18] M. T. Hansen and S. R. Sharpe, Relativistic, modelindependent, three-particle quantization condition, Phys. Rev. D 90, 116003 (2014).

[19] M. T. Hansen and S. R. Sharpe, Expressing the threeparticle finite-volume spectrum in terms of the three-tothree scattering amplitude, Phys. Rev. D 92, 114509 (2015).

[20] H.-W. Hammer, J.-Y. Pang, and A. Rusetsky, Three-particle quantization condition in a finite volume: 1 . The role of the three-particle force, J. High Energy Phys. 09 (2017) 109.
[21] H. W. Hammer, J. Y. Pang, and A. Rusetsky, Three particle quantization condition in a finite volume: 2. General formalism and the analysis of data, J. High Energy Phys. 10 (2017) 115.

[22] M. Mai and M. Döring, Three-body unitarity in the finite volume, Eur. Phys. J. A 53, 240 (2017).

[23] T. D. Blanton and S. R. Sharpe, Equivalence of relativistic three-particle quantization conditions, Phys. Rev. D $\mathbf{1 0 2}$, 054515 (2020).

[24] F. Romero-López, A. Rusetsky, N. Schlage, and C. Urbach, Relativistic $N$-particle energy shift in finite volume, J. High Energy Phys. 02 (2021) 060.

[25] M. T. Hansen and S. R. Sharpe, Lattice QCD and threeparticle decays of resonances, Annu. Rev. Nucl. Part. Sci. 69, 65 (2019).

[26] M. Mai, M. Döring, and A. Rusetsky, Multi-particle systems on the lattice and chiral extrapolations: A brief review, Eur. Phys. J. Special Topics 230, 1623 (2021).

[27] R. Mertig, M. Bohm, and A. Denner, FEYN CALC: Computer algebraic calculation of Feynman amplitudes, Comput. Phys. Commun. 64, 345 (1991).

[28] V. Shtabovenko, R. Mertig, and F. Orellana, New developments in FeynCalc 9.0, Comput. Phys. Commun. 207, 432 (2016).

[29] V. Shtabovenko, R. Mertig, and F. Orellana, FeynCalc 9.3: New features and improvements, Comput. Phys. Commun. 256, 107478 (2020).

[30] J. A. M. Vermaseren, New features of FORM, arXiv:math$\mathrm{ph} / 0010025$.

[31] G. J. van Oldenborgh and J. A. M. Vermaseren, New algorithms for one loop integrals, Z. Phys. C 46, 425 (1990).

[32] T. Hahn and M. Perez-Victoria, Automatized one-loop calculations in four and $D$ dimensions, Comput. Phys. Commun. 118, 153 (1999).

[33] S. R. Coleman, J. Wess, and B. Zumino, Structure of phenomenological Lagrangians. 1., Phys. Rev. 177, 2239 (1969).

[34] S. Weinberg, Nonlinear realizations of chiral symmetry, Phys. Rev. 166, 1568 (1968).

[35] J. Bijnens, G. Colangelo, G. Ecker, J. Gasser, and M. E. Sainio, Elastic $\pi \pi$ scattering to two loops, Phys. Lett. B 374, 210 (1996).

[36] J. Bijnens, G. Colangelo, G. Ecker, J. Gasser, and M. E. Sainio, Pion-pion scattering at low energy, Nucl. Phys. B508, 263 (1997); B517, 639(E) (1998).

[37] A. Dobado and J. Morales, Pion mass effects in the large N limit of chiral perturbation theory, Phys. Rev. D 52, 2878 (1995).

[38] J. Bijnens and L. Carloni, Leading logarithms in the massive $O(N)$ nonlinear sigma model, Nucl. Phys. B827, 237 (2010).

[39] J. Bijnens and L. Carloni, The massive $O(N)$ non-linear sigma model at high orders, Nucl. Phys. B843, 55 (2011).

[40] J. Bijnens and G. Ecker, Mesonic low-energy constants, Annu. Rev. Nucl. Part. Sci. 64, 149 (2014).

[41] G. Colangelo, J. Gasser, and H. Leutwyler, $\pi \pi$ scattering, Nucl. Phys. B603, 125 (2001).

[42] S. Aoki et al., Review of lattice results concerning lowenergy particle physics, Eur. Phys. J. C 77, 112 (2017). 